BMJ Open

Diabetes

Research

\& Care

\title{
Adherence to lipid-lowering therapy and risk for cardiovascular disease and death in type 1 diabetes mellitus: a population-based study from the Swedish National Diabetes Register
}

\author{
Christel Hero (1) , ${ }^{1,2}$ Sofia Axia Karlsson (1) , ${ }^{3}$ Stefan Franzén, ${ }^{4,5}$ \\ Ann-Marie Svensson, ${ }^{1,4}$ Mervete Miftaraj, ${ }^{4}$ Soffia Gudbjörnsdottir, ${ }^{1,4}$ \\ Karolina Andersson Sundell, ${ }^{3,6}$ Björn Eliasson, ${ }^{1,2}$ Katarina Eeg-Olofsson (1) 1,2
}

\begin{abstract}
To cite: Hero C, Karlsson SA, Franzén S, et al. Adherence to lipid-lowering therapy and risk for cardiovascular disease and death in type 1 diabetes mellitus: a population-based study from the Swedish National Diabetes Register. BMJ Open Diab Res Care 2020;8:e000719. doi:10.1136/ bmjdrc-2019-000719
\end{abstract}

\section{- Additional material is published online only. To view please visit the journal online (http://dx.doi.org/10.1136/ bmjdrc-2019-000719).}

Received 27 June 2019 Revised 4 November 2019 Accepted 22 November 2019
Check for updates

\section{(C) Author(s) (or their} employer(s)) 2020. Re-use permitted under CC BY-NC. No commercial re-use. See rights and permissions. Published by BMJ.

For numbered affiliations see end of article.

Correspondence to Dr Christel Hero; christel.hero@vgregion.se

\section{ABSTRACT}

Aims/hypothesis Dyslipidemia is an important modifiable risk factor and lipid-lowering treatment (LLT) is essential to reduce the risk of cardiovascular disease (CVD). Studies in type 2 diabetes indicate that low adherence to statin therapy is a barrier to reach full protective potential, and less is known in type 1 diabetes (T1D). The aim was to assess risk of CVD by adherence and nonpersistence to LLT in T1D.

Method A population-based study with a retrospective longitudinal design was conducted between 2006 and 2010, with follow-up until December 2013. In total, 6192 adult individuals with T1D, initiating LLT between 2006 and 2010, were included. Information on LLT, socioeconomic characteristics, comorbidities and cardiovascular events were collected. After 18 months, refill adherence was estimated by calculating medication possession ratio (MPR). Nonpersistence was defined as being without medicines on hand for at least 180 days. Individuals were thereafter followed until CVD, death or end of follow-up in December 2013. Cox regression analyses were performed to assess adherence level and nonpersistence of LLT as predictor of CVD. Analyses were adjusted for cardiovascular risk factors and socioeconomic status.

Results Mean MPR was $72 \%, 52 \%$ of the participants had an MPR above $80 \%$ and $27 \%$ discontinued LLT. There were 637 nonfatal and 58 fatal CVD events, mean follow-up 3.6 and 3.9 years, respectively. MPR above $80 \%$ was associated with reduced risk for nonfatal CVD compared with lower MPR, HR 0.78 (95\% Cl 0.65 to 0.93$)$ ). For fatal CVD, results indicated a negative effect of high adherence but the association did not reach statistical significance, HR 1.96 (0.96 to 4.01). Individuals discontinuing LLT had higher risk of nonfatal CVD, HR 1.43 (95\% Cl 1.18 to 1.73).

Conclusions/Interpretation In T1D, the risk for nonfatal CVD was lower among individuals with high adherence and higher among those discontinuing LLT within 18 months. It is important to evaluate and emphasize adherence to prescribed LLT at clinical visits to achieve treatment goals and reduce the risk of CVD.

\section{Significance of this study}

What is already known about this subject?

- Cardiovascular disease (CVD) is the leading cause of shorter life expectancy in type 1 diabetes.

- Dyslipidemia is an important modifiable risk factor and lipid-lowering treatment is essential to reduce the risk of CVD.

- Studies in general population and in type 2 diabetes indicate that low adherence to statin therapy is a barrier to reach full protective potential.

What are the new findings?

- In individuals with type 1 diabetes initiating lipidlowering therapy, high adherence to treatment was associated with $22 \%$ lower risk of nonfatal CVD.

- Discontinuing the lipid-lowering therapy led to a $43 \%$ higher risk for nonfatal CVD.

How might these results change the focus of research or clinical practice?

- It is important to evaluate and emphasize adherence to prescribed lipid-lowering therapy in clinical practice to achieve treatment goals and reduce the risk of CVD in individuals with type 1 diabetes.

\section{INTRODUCTION}

Despite positive trends in the latest decades, cardiovascular disease (CVD) is still the leading cause of shorter life expectancy in individuals with type 1 diabetes. ${ }^{1-3}$ Effects of hyperglycemia and nephropathy and also traditional risk factors such as smoking, hypertension, obesity and dyslipidemia are considered important for the excess of cardiovascular morbidity and mortality seen in type 1 diabetes. $^{45}$

Lipid-lowering treatment (LLT) with HMGCoA reductase inhibitors (statins) has in a multitude of studies proved to prevent major 
cardiovascular events and death, in the general population as well as in patients with diabetes. ${ }^{67}$ Cholesterol Treatment Trialists' Collaborators meta-analysis of statins in 18686 subjects with diabetes, the majority having type 2 diabetes, demonstrated a $21 \%$ reduction in major cardiovascular events for each $1.0 \mathrm{mmol} / \mathrm{L}(38.7 \mathrm{mg} / \mathrm{dL})$ reduction in low-density lipoprotein (LDL) cholesterol. ${ }^{8}$ In an observational study from the Swedish National Diabetes Register (NDR), we could show a risk reduction of $22 \%-44 \%$ for CVD and death also in individuals with type 1 diabetes on LLT in primary prevention. ${ }^{9}$

In 2003, the WHO launched a report on adherence to medication for chronic diseases and concluded that poor adherence have large clinical impacts on health outcomes and utilization of health services worldwide. ${ }^{10}$ Chowdury et al published a meta-analysis in 2013 and calculated that approximately $9 \%$ of CVD events in Europe could be attributed to poor adherence to cardiovascular medication alone. ${ }^{11}$ Due to poor adherence, the effects of lipidlowering medication seen in the context of a randomized controlled trial may be reduced in a real world diabetes care. ${ }^{12}$ Studies in the general population have shown an incremental improvement in clinical outcomes with the higher the adherence and the longer the persistence to LLT. ${ }^{1314}$ In studies on patients with diabetes, the majority with type 2 diabetes, nonadherence to LLT have been associated with an increased risk for CVD and death both in primary and secondary prevention. ${ }^{15} 16$ Less is known in type 1 diabetes.

The aim of this study was to assess refill adherence to LLT and nonpersistence (ie, discontinuation) of LLT in individuals with type 1 diabetes in relation to cardiovascular outcomes and death. We hypothesized that high adherence and persistence to LLT in individuals with type 1 diabetes would be associated with a lower risk of CVD.

\section{RESEARCH DESIGN AND METHODS \\ Study population}

We included individuals, 18 years or older, with type 1 diabetes in the Swedish NDR and who initiated use of LLT between 1 July 2006 and 31 December 2010. Type 1 diabetes was defined on the basis of epidemiological data: a diagnosis at the age of 30 years or younger and treatment with insulin alone. All patients were followed during the first 18 months on LLT until the first day of multidose dispensed medicines, death or migration, leading to censoring from the study. Individuals experiencing a nonfatal cardiovascular event during the 18-month exposure assessment period remained in the study. For the 18 months since initiation of LLT, we measured refill adherence to medication and time to discontinuation for each individual and thereafter followed them until a first cardiovascular event or until end of follow-up 31 December 2013. Events of all-cause death were followed until end of May 2015.
Patients, registered in NDR, with type 1 diabetes who filled at least one prescription for lipid-lowering medicines (except bile acid sequestrants) between 1 July 2006 and 31 December 2010 $(n=16,864)$

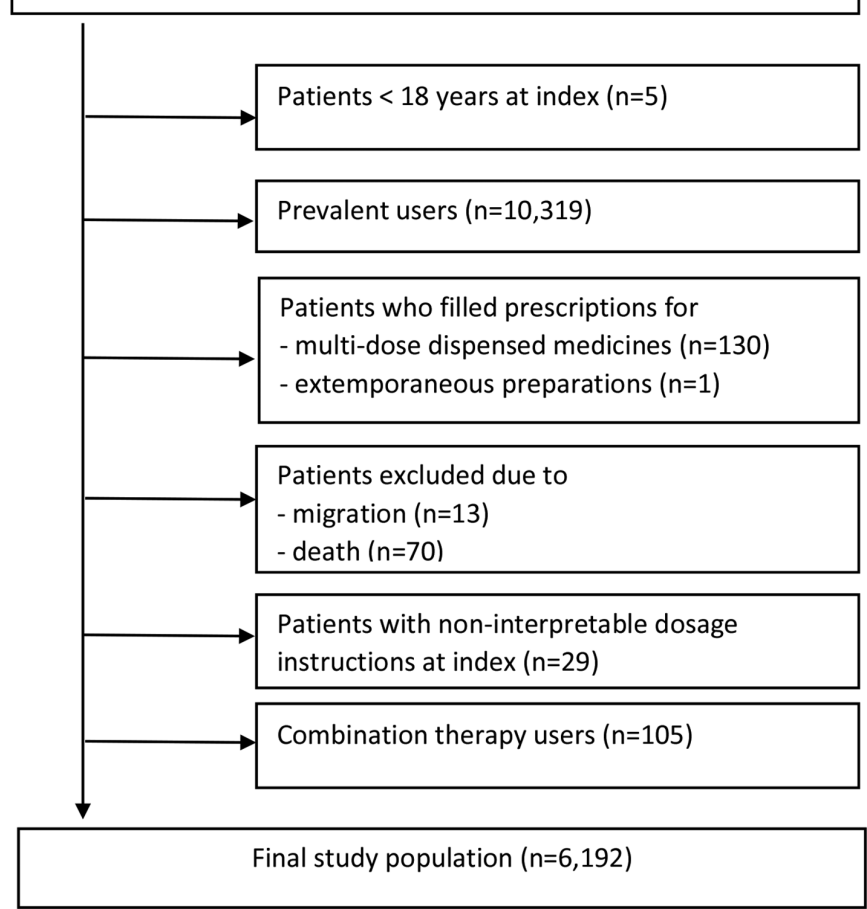

Figure 1 Flowchart for inclusion and exclusion criteria for the study population. NDR, National Diabetes Register.

To identify novel users of LLT, we excluded individuals who filled a prescription of LLT within 365 days prior to inclusion, that is, prevalent users. See figure 1 for flowchart. Individuals were also excluded if they filled any prescriptions for lipid-lowering extemporaneous preparations, prescriptions lacking information of package size or monotherapy of bile acid sequestrants, the latter often prescribed for other indications than hyperlipidemia. ${ }^{17}$ We also excluded combination therapy users, unless bile sequestrants, if more than one substance or strength of the same substance were dispensed on the same date or if a previously filled substance or strength was filled once more within 45 days after the previous supply ended and another substance or strength was filled in between. Products containing multiple lipid-lowering substances in the same unit were considered monotherapy.

\section{Measurements of adherence and discontinuation}

In the present study, we have investigated refill adherence and measured the medication possession ratio (MPR), that is, the proportion of days with medicines on hand according to filled prescriptions, during the first observation period of 18 months. The refill adherence is considered one of the most reliable objective measures of adherence in large patient groups with long-term treatments of chronic conditions. ${ }^{18}$ We used 18 months of exposure as recommended by a previous study of statins for the general Swedish population, giving us 
robust data on adherence with the possibility of at least six refills, since most prescriptions of long-term medication are commonly issued for 1 year treatment, with 3-month dispensation intervals. ${ }^{19}$ Using 18 months of exposure rendered enough follow-up time for evaluation of events related to adherence and discontinuation of therapy.

MPR was measured both as a continuous variable and as dichotomized with a cut-off value $>80 \%$ defined as high adherence and $\leq 80 \%$ as low adherence. An $80 \%$ cutoff has commonly been used in other studies assessing adherence and is also the level above where the cardioprotective benefits of LLT, mainly statins, become obvious. ${ }^{20}{ }^{21}$ We defined persistence as the length of continuous use from initiation to discontinuation of LLT. Patients who discontinued treatment within the first 18 months were considered nonpersistent to LLT. Discontinuation was defined as a gap of at least 180 days between two filled prescriptions for LLT, representing two refills within the Swedish reimbursement system. For these measurements, we used an algorithm defined in a previous study investigating adherence in individuals with type 2 diabetes. ${ }^{22}$

\section{Registries used in the study}

Clinical baseline characteristics were retrieved from the NDR. NDR was initiated in 1996 as a tool for local quality assurance and as a feedback tool in diabetes care. ${ }^{23}$ Each patient provides informed consent. Roughly 98\% of all individuals age 18 and older with type 1 diabetes in Sweden are included in the register.

The Swedish Prescribed Drug Register (SPDR) provided information on age, sex, type of medicine, package size, date of dispensing and free text dosage instructions from the prescriber. The SPDR has since July 1, 2005 individualized its data on all prescriptions filled in Sweden and has been characterized in an earlier study. ${ }^{24}$

The unique Swedish personal identity number further made it possible to link register data from the Swedish National Patient Register, the Cause of Death Register (both administered by the Swedish National Board of Health and Welfare), as well as the Longitudinal Integration Database for Health Insurance and Labour Market Studies (LISA, administered by Statistics Sweden). The NPR has nationwide coverage since 1987 and includes mandatory information on all principal and secondary hospital discharge diagnoses classifying them by The International Classification Disease (ICD) system; we used ICD-10 with documented discharge diagnoses since 1997. Sensitivity and specificity for diagnoses of acute myocardial infarction, coronary heart disease, hospitalization for heart failure, atrial fibrillation and stroke have been validated. ${ }^{25}{ }^{26}$ From LISA, we obtained data on socioeconomic status ${ }^{27}$ and from the Cause of Death Register date and cause of death.

The study was approved by the Regional Ethics Review Board at the University of Gothenburg, Gothenburg, Sweden.

\section{Examinations at baseline}

Clinical data from the NDR: Data were collected before index date up to 2 years prior to inclusion with last observation carried forward (LOCF). LDL-cholesterol values were calculated using Friedewald's formula if triglycerides $<4.0 \mathrm{mmol} / \mathrm{L}^{28}$ Microalbuminuria was defined as albumin/creatinine ratio $3-30 \mathrm{mg} / \mathrm{mmol}$ or U-albumin $20-200 \mu \mathrm{g} / \mathrm{min}$ or $20-300 \mathrm{mg} / \mathrm{L}$ and macroalbuminuria as albumin/creatinine ratio $>30 \mathrm{mg} / \mathrm{mmol}$ or U-albumin $>200 \mu \mathrm{g} / \mathrm{min}$ or $>300 \mathrm{mg} / \mathrm{L}$. Glomerular filtration rate was estimated with the MDRD (modification of diet in renal disease) equation. ${ }^{29}$ Smoking was coded as present if the patient was a current smoker. Physical activity was categorized into low or high level where low exercise meant to never exercise or to exercise less than 1 time per week while high meant exercise more than 1-2 times per week.

Socioeconomic status from LISA: Data on disposable individual income in hundred Swedish kronor (latest annual income, not adjusted for inflation), highest educational level, country of birth and marital status were retrieved from the register \pm 1 year from index date using the closest value. Education was stratified into compulsory school or lower ( $\leq 9$ years), upper secondary school (10-12 years) and postsecondary (college/university). Immigrant status was defined as Swedish native or immigrant, depending on country of birth. Marital categories were single, married, divorced or widowed.

Comorbidities and events: Collected before baseline examination and during follow-up by linking data from the NDR to the Swedish National Patient Register and the Causes of Death Register. Information on prior comorbidities was retrieved before index back to 1997 with LOCF. The following comorbidities and events were assessed: myocardial infarction (ICD-10 code I21), unstable angina (I20.0), percutaneous coronary intervention (PCI) and/or coronary artery bypass grafting (CABG), coronary heart disease (I20-I25), stroke defined as cerebral infarction, intracerebral hemorrhage or unspecified stroke (I61, I63, I64), peripheral vascular disease (PVD) defined as peripheral atherosclerosis in the arteries of the extremities (I70.2, I73.9, I79.2) or diabetes mellitus with complications in the peripheral arteries (E10.5, E11.5, E14.5), endovascular intervention in the peripheral arteries and/or peripheral artery bypass grafting, atrial fibrillation (I48), history of congestive heart failure (I50) and any cancer (C00-C97). For events, we evaluated total mortality and nonfatal or fatal CVD and a composite of nonfatal and fatal CVD. In the cox regression analysis a CVD event was a composite of myocardial infarction, unstable angina, PCI, CABG, stroke, PVD, endovascular interventions and/or peripheral artery by-pass grafting. In the spline analysis a nonfatal CVD was a composite of unstable angina, myocardial infarction, stroke and PVD.

Missing data: For clinical characteristics there were 21\% missing data, varying between variables, from no missing on gender to $17 \%$ missing on HbAlc and 33\% missing 
for information on physical activity. For socioeconomic variables, only $0.6 \%$ were missing information on education and $0.1 \%$ on marital status and income respectively. There were no missing data on comorbidities.

\section{Statistical analyses}

The observed data are described using standard descriptive statistics such as mean, SD, counts and percentages. A Cox proportional hazard regression analysis with HRs with 95\% CI was performed to analyze MPR as predictor of nonfatal and fatal CVD and all-cause death, comparing the group with MPR $>80 \%$ to the group with $\mathrm{MPR} \leq 80 \%$ and also comparing discontinuers versus continuers of LLT in relation to risk for a cardiovascular event or death. We also performed a sensitivity analysis with a Cox regression analysis as above but excluding the patients having a nonfatal CVD event during the exposure assessment period. The Cox regression analyses were adjusted for traditional risk factors, comorbidities and socioeconomic status as presented in table 1. Missing data were handled by means of multiple imputation. We used multiple imputation by chained equations algorithm imputing 10 complete datasets. Separate analyses were run on each imputed data set and the results were combined using Rubin's rules.

The impact of MPR was further investigated by fitting a Cox regression model where the effect of MPR as a continuous measure was modeled by a smoothing spline with $7 \mathrm{df}$ to one of the imputed data sets, adjusted for age, sex and previous CVD. All hypothesis tests were evaluated using a $5 \%$ significance level without any adjustment for multiple comparisons. The analysis was performed using SAS 9.4 and R 3.4.3.

\section{RESULTS}

Table 2 presents baseline characteristics and adherence data for all 6192 participants by high and low adherence and also discontinuers and continuers as a measure of persistence. In summary, mean age was 45 years, diabetes duration 29 years and 58\% were of male gender. Fortythree per cent had antihypertensive medication. Previous CVD was present in $9 \%$ of the participants. Ninety-three per cent were born in Sweden. Forty-five per cent were married and $12 \%$ divorced. Seventeen per cent had an education of 9 years or less, $29 \%$ had gone to college or university.

The mean MPR over 18 months was $72 \% \pm 28 \%$ (median $83 \%), 52 \%$ of the participants had an MPR $>80 \%$ (median 97\%) and 27\% discontinued with LLT during the 18-month exposure assessment period. In the group with high adherence, the frequency of individuals with previous CVD and with concurrent medication (antihypertensives and anticoagulants) was higher. Smokers were less adherent than nonsmokers. The majority of the individuals, $99 \%$, were on treatment with statins, simvastatin being the most common at $94.4 \%$, followed by atorvastatin at $3.4 \%$ (see online supplementary table 1 ).

Table 1 shows number of events and fully adjusted HRs. There was a total of 637 nonfatal CVD events, 58 fatal

Table 1 Number of events and adjusted HRs $(95 \% \mathrm{Cl})$ for MPR as a continuous measure (MPR 18 months) per one unit increase and for high refill adherence (MPR $>80 \%$ ) with MPR $>80 \%$ compared with MPR $\leq 80 \%$ and discontinuers* ${ }^{*}$ compared with continuers after 18 months

\begin{tabular}{|c|c|c|c|c|}
\hline Event & $\begin{array}{l}\text { Number of } \\
\text { events (n) }\end{array}$ & Exposure & HR $(95 \% \mathrm{Cl})$ & $P$ value \\
\hline \multirow[t]{3}{*}{ Nonfatal CVD } & 637 & MPR 18 months & 0.99 (0.99 to 1.00$)$ & 0.0001 \\
\hline & & MPR $>80 \%$ & 0.78 (0.65 to 0.93$)$ & 0.005 \\
\hline & & Discontinuation 18 months & 1.43 (1.18 to 1.73$)$ & 0.0003 \\
\hline \multirow[t]{3}{*}{ Nonfatal/fatal CVD } & 695 & MPR 18 months & 0.99 (0.99 to 1.00$)$ & 0.0001 \\
\hline & & MPR $>80 \%$ & 0.79 (0.66 to 0.94$)$ & 0.01 \\
\hline & & Discontinuation 18 months & $1.47(1.21$ to 1.78$)$ & 0.0001 \\
\hline \multirow[t]{3}{*}{ CVD death } & 58 & MPR 18 months & 1.01 (1.00 to 1.02$)$ & 0.23 \\
\hline & & MPR $>80 \%$ & 1.96 (0.96 to 4.01$)$ & 0.06 \\
\hline & & Discontinuation 18 months & 0.88 (0.41 to 1.89$)$ & 0.74 \\
\hline \multirow[t]{3}{*}{ Total death } & 302 & MPR 18 months & 1.00 (1.00 to 1.00$)$ & 0.74 \\
\hline & & $\mathrm{MPR}>80 \%$ & $0.91(0.71$ to 1.18$)$ & 0.49 \\
\hline & & Discontinuation 18 months & 1.17 (0.85 to 1.60$)$ & 0.33 \\
\hline
\end{tabular}

Adjusted for age, gender, diabetes duration, BMI, smoking, physical activity, HbA1c, systolic and diastolic blood pressure, LDL-cholesterol and HDL-cholesterol, triglycerides, albuminuria, eGFR, use of insulin pump, antihypertensive treatment, previous CVD (including coronary artery bypass grafting, percutaneous coronary intervention and endovascular grafting), atrial fibrillation, heart failure, cancer and socioeconomic status (income, marital status, education and country of origin as presented in table 2). Analyses were based on 10 imputations.

${ }^{*}$ Discontinuation (ie, nonpersistence) defined as being without medicines on hand for $>180$ days.

BMI, body mass index; CVD, cardiovascular disease; eGFR, estimated glomerular filtration rate; HbA1c, glycated hemoglobin A1c; HDL, high-density lipoprotein; LDL, low-density lipoprotein; MPR, medication possession ratio. 
Table 2 Baseline characteristics and adherence data for the study population; in all, in individuals with MPR $\leq 80$ and $>80 \%$ and in discontinuers and continuers of LLT at index and 18 months after initiation of LLT

\begin{tabular}{|c|c|c|c|c|c|}
\hline \multirow[b]{2}{*}{ Variables } & \multirow[b]{2}{*}{$\begin{array}{l}\text { All } \\
n=6192\end{array}$} & \multicolumn{4}{|c|}{ After 18 months of LLT } \\
\hline & & $\begin{array}{l}\text { MPR } \leq 80 \% \\
n=2970\end{array}$ & $\begin{array}{l}M P R>80 \% \\
n=3222\end{array}$ & $\begin{array}{l}\text { Discontinuers } \\
n=1653\end{array}$ & $\begin{array}{l}\text { Continuers } \\
n=4539\end{array}$ \\
\hline \multicolumn{6}{|l|}{ Patient characteristics } \\
\hline Age (years) & $45 \pm 12^{*}$ & $43 \pm 12$ & $47 \pm 12$ & $42 \pm 12$ & $46 \pm 12$ \\
\hline Gender: male & $3559(58)^{*}$ & $1785(60)$ & $1774(55)$ & $1012(61)$ & $2547(56)$ \\
\hline Diabetes duration (years) & $29 \pm 14$ & $27 \pm 13$ & $31 \pm 14$ & $26 \pm 13$ & $27 \pm 13$ \\
\hline $\mathrm{BMI}\left(\mathrm{kg} / \mathrm{m}^{2}\right)$ & $26.3 \pm 4.2$ & $26.4 \pm 4.2$ & $26.3 \pm 4.2$ & $26.3 \pm 4.3$ & $26.4 \pm 4.2$ \\
\hline Smokers & $651(13)$ & $352(15)$ & $299(11)$ & $209(17)$ & $442(12)$ \\
\hline Low physical activity $\dagger$ & $891(22)$ & $430(22)$ & $61(21)$ & $253(24)$ & $638(21)$ \\
\hline \multicolumn{6}{|l|}{ Biomarkers } \\
\hline $\mathrm{HbA} 1 \mathrm{c}(\mathrm{mmol} / \mathrm{mol})$ & $66 \pm 14$ & $67 \pm 14$ & $64 \pm 13$ & $68 \pm 14$ & $65 \pm 13$ \\
\hline HbA1c NGSP (\%) & $8.1 \pm 3.4$ & $8.3 \pm 3.4$ & $8.0 \pm 3.4$ & $8.3 \pm 3.5$ & $8.1 \pm 3.4$ \\
\hline Systolic blood pressure $(\mathrm{mm} \mathrm{Hg})$ & $130 \pm 16$ & $129 \pm 16$ & $130 \pm 15$ & $129 \pm 16$ & $130 \pm 16$ \\
\hline Diastolic blood pressure $(\mathrm{mm} \mathrm{Hg})$ & $75 \pm 9$ & $75 \pm 9$ & $74 \pm 9$ & $75 \pm 9$ & $74 \pm 9$ \\
\hline LDL-cholesterol (mmol/L) & $3.3 \pm 0.8$ & $3.4 \pm 0.8$ & $3.2 \pm 0.7$ & $3.4 \pm 0.8$ & $3.3 \pm 0.7$ \\
\hline HDL-cholesterol (mmol/L) & $1.6 \pm 0.5$ & $1.6 \pm 0.5$ & $1.6 \pm 0.5$ & $1.6 \pm 0.5$ & $1.6 \pm 0.5$ \\
\hline Triglycerides (mmol/L) & $1.2 \pm 0.9$ & $1.3 \pm 0.9$ & $1.2 \pm 0.8$ & $1.3 \pm 0.9$ & $1.2 \pm 0.8$ \\
\hline eGFR (mL/min) & $91 \pm 25$ & $93 \pm 25$ & $89 \pm 24$ & $94 \pm 26$ & $90 \pm 24$ \\
\hline Microalbuminuria & $824(18)$ & $385(18)$ & $439(18)$ & $218(19)$ & $606(18)$ \\
\hline Macroalbuminuria & $369(7.7)$ & $164(7.4)$ & $205(8.0)$ & $84(7.0)$ & $285(7.9)$ \\
\hline \multicolumn{6}{|l|}{ Socioeconomic status } \\
\hline Disposable individual income & $2145(3797)$ & 2089 (1572) & $2197(5042)$ & 2042(1794) & $2183(4300)$ \\
\hline Married & $2810(45)$ & $1230(42)$ & $1580(49)$ & $653(40)$ & $2157(48)$ \\
\hline Single & $2509(41)$ & $1313(44)$ & $1196(37)$ & $759(46)$ & $1750(39)$ \\
\hline Divorced & $774(13)$ & $383(13)$ & $391(12)$ & $221(13)$ & $553(12)$ \\
\hline Widowed & $91(1.5)$ & $39(1.3)$ & $52(1.6)$ & $17(1.0)$ & $74(1.6)$ \\
\hline Education 9 years or less & $1050(17)$ & $490(17)$ & $560(18)$ & $275(17)$ & $775(17)$ \\
\hline Education $10-12$ years & $3314(54)$ & $1639(56)$ & $1675(52)$ & $930(57)$ & $2384(53)$ \\
\hline College/university & $1792(29)$ & $824(28)$ & $968(30)$ & $435(27)$ & $1357(30)$ \\
\hline Born in Sweden & $5752(93)$ & $2736(92)$ & $3016(94)$ & $1503(91)$ & $4249(94)$ \\
\hline \multicolumn{6}{|l|}{ Treatment } \\
\hline Multiple daily injection & 3891 (84) & $1825(83)$ & $2066(84)$ & $1005(84)$ & $2886(84)$ \\
\hline Insulin pump therapy & $761(16)$ & $366(17)$ & $395(16)$ & $194(16)$ & $567(16)$ \\
\hline Antihypertensive medication & $2642(43)$ & $992(33)$ & $1650(51)$ & $526(32)$ & $2116(47)$ \\
\hline Anticoagulants (excluding ASA) & $183(3.0)$ & $69(2.3)$ & $114(3.5)$ & $42(2.5)$ & $141(3.1)$ \\
\hline ASA & $982(16)$ & $356(12)$ & $626(19)$ & $178(11)$ & $804(18)$ \\
\hline \multicolumn{6}{|l|}{ Previous disease } \\
\hline Unstable angina & $168(2.7)$ & $64(2.2)$ & $104(3.2)$ & $40(2.4)$ & $128(2.8)$ \\
\hline Acute myocardial infarction & $150(2.4)$ & $40(1.3)$ & $110(3.4)$ & $24(1.5)$ & $126(2.8)$ \\
\hline Ischemic disease & $102(1.6)$ & $38(1.3)$ & $64(2.0)$ & $29(1.8)$ & $73(1.6)$ \\
\hline Stroke & $113(1.8)$ & $40(1.3)$ & $73(2.3)$ & $20(1.2)$ & $93(2.0)$ \\
\hline Peripheral vascular disease & $211(3.4)$ & $86(2.9)$ & 125 (3.9) & $57(3.4)$ & $154(3.4)$ \\
\hline Cardiovascular disease & $559(9.0)$ & $201(6.8)$ & $358(11.1)$ & $124(7.5)$ & 435 (9.6) \\
\hline Coronary artery bypass grafting & $58(0.9)$ & $18(0.6)$ & $40(1.2)$ & $14(0.8)$ & $44(1.0)$ \\
\hline
\end{tabular}


Table 2 Continued

\begin{tabular}{|c|c|c|c|c|c|}
\hline \multirow[b]{2}{*}{ Variables } & \multirow[b]{2}{*}{$\begin{array}{l}\text { All } \\
n=6192\end{array}$} & \multicolumn{4}{|c|}{ After 18 months of LLT } \\
\hline & & $\begin{array}{l}\mathrm{MPR} \leq 80 \% \\
\mathrm{n}=2970\end{array}$ & $\begin{array}{l}\text { MPR>80\% } \\
n=3222\end{array}$ & $\begin{array}{l}\text { Discontinuers } \\
n=1653\end{array}$ & $\begin{array}{l}\text { Continuers } \\
n=4539\end{array}$ \\
\hline Percutaneous coronary intervention & $109(1.8)$ & $29(1.0)$ & $80(2.5)$ & $18(1.1)$ & $91(2.0)$ \\
\hline Heart failure & $69(1.1)$ & $17(0.6)$ & $52(1.6)$ & $10(0.6)$ & $59(1.3)$ \\
\hline Atrial fibrillation & $48(0.8)$ & $25(0.8)$ & $23(0.7)$ & $18(1.1)$ & $30(0.7)$ \\
\hline Cancer & $80(1.3)$ & $30(1.0)$ & $50(1.6)$ & $19(1.1)$ & $61(1.3)$ \\
\hline \multicolumn{6}{|l|}{ Adherence } \\
\hline MPR mean (SD) & $72(28)$ & $48(22)$ & $95(6)$ & $33(16)$ & $87(14)$ \\
\hline MPR median (IQR) & 83 & $54(33 ; 70)$ & $97(91 ; 100)$ & $36(18 ; 45)$ & $92(77 ; 99)$ \\
\hline
\end{tabular}

${ }^{*}$ Data are presented as means \pm SD for continuous variables, and $\mathrm{N}(\%)$ for categorical variables. MPR also as median with interquartile range (IQR)

†Low physical activity, never or exercise less than one time per week.

ASA; acetylsalicylic acid; BMI, body mass index; eGFR, estimated glomerular filtration rate; HbA1c, glycated hemoglobin (A1c); HDL, high-density lipoprotein; LDL, low-density lipoprotein; LLT, lipid-lowering therapy; MPR, medication possession ratio; NGSP, National Glycohemoglobin Standardization Program.

CVD events and 302 deaths for other causes during mean follow-up 3.6 years for nonfatal events and 3.9 years for fatal events. Patients with a high adherence (MPR $>80 \%$ ) had a 22\% lower risk of nonfatal CVD compared with patients with MPR $\leq 80 \%$. Patients discontinuing LLT had a $43 \%$ higher risk of nonfatal CVD. For those with an MPR $>80 \%$, an association to increased risk of fatal CVD HR 1.96 (0.96 to 4.01) was found, but it did not reach statistical significance $(p=0.06)$. For the composite of nonfatal and fatal CVD, there was a $21 \%$ significantly reduced risk with high adherence to LLT. There was no association between adherence or discontinuation and total death.

In the extra analysis excluding the individuals that had a nonfatal cardiovascular event during the exposure assessment period, the point estimate for fatal CVD changed only marginally (MPR 18 months $>80 \% 1.98$ (0.86 to 4.52 ), $\mathrm{p}=0.11$ ).

The relative rate of nonfatal CVD $(95 \% \mathrm{CI})$ in relation to MPR as a continuous measure is illustrated in figure 2. The cubic spline shows higher CVD risk with lower MPR with the highest risk in those with an MPR from $20 \%$ to $40 \%$ and the lowest risk in those with an MPR above $80 \%$.

\section{DISCUSSION}

This observational nationwide study in 6192 individuals with type 1 diabetes was designed to assess refill adherence to lipid-lowering therapy (LLT) in a real-life setting and also to evaluate adherence in relation to the risk of a cardiovascular event, fatal or nonfatal and all-cause death. Our results show a significant effect of high refill adherence with a 22\% reduced risk of developing a nonfatal cardiovascular event for the individuals with an MPR $>80 \%$ compared with those with a an MPR $\leq 80 \%$. In individuals discontinuing therapy during 18 months of exposure, there was a significantly increased risk of $43 \%$ for a nonfatal cardiovascular event. For the composite of nonfatal and fatal CVD, we observed a $21 \%$ significantly reduced risk with high adherence to LLT.

Adherence to medication is important to gain full potential of LLT and reach the results of primary and secondary prevention with LLT seen in randomized controlled trials. In a systematic review of 19 journal articles from 1999 to 2009 on adherence to statins and its impact on outcome, Simpson et al concluded that high levels of adherence were associated with significant reductions in a range of outcomes such as fatal and nonfatal CVD as well as all-cause death and hospitalizations. ${ }^{13}$ Deshpande et al could confirm these findings in a more recent review published 2017, assessing 84 realworld studies on adherence and persistence from 2006 to $2016 .^{21}$

The effect of MPR 18 months on non fatal CVD

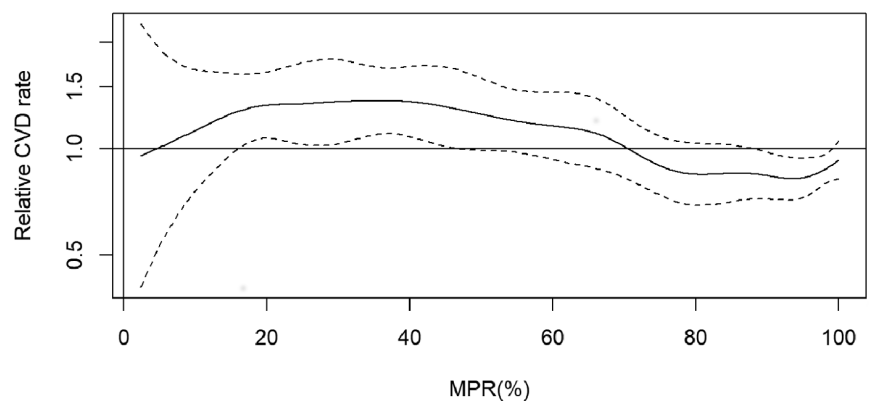

Figure 2 The effect of MPR calculated over 18 months on risk of nonfatal CVD. Relative CVD rate with 95\% Cls adjusted for age, sex and previous CVD (including coronary artery bypass grafting, percutaneous coronary interventions and endovascular grafting). Nonfatal CVD: composite of unstable angina, myocardial infarction, stroke, peripheral vascular disease, coronary artery bypass grafting, percutaneous coronary intervention and endovascular grafting. CVD, cardiovascular disease; MPR, medication possession ratio. 
Many studies show, unlike our study, a significant effect of high adherence on cardiovascular death and all-cause death. ${ }^{30}$ In our study, the analysis for fatal CVD showed an association with high adherence to LLT, however nonsignificant. The number of fatal cardiovascular events were few and when analyzing the composite of nonfatal and fatal CVD, the association was significantly positive for high adherence to LLT. Since individuals experiencing a CVD event during the exposure assessment period remained in the study, both adherence and outcome could be affected. One could expect a higher adherence to LLT directly adjacent to an event and also a higher risk for a new event in the near future. However, the sensitivity analysis of those with no event during the exposure assessment period showed only marginal effects on the estimates. Hence, the unexpected observation for CVD death is likely to be biased and due to residual confounding, unmeasured or inherited by the study design.

The evidence for the efficacy of LLT in prevention of CVD in diabetes is undisputed. ${ }^{832} 33$ Poor adherence to cardiovascular medication alone account for approximately $9 \%$ of CVD events in the general population. ${ }^{11}$ In our study, only $52 \%$ of the participants had an MPR above $80 \%$ after 18 months. This level of adherence is consistent with the adherence measures in a review by Cramer et $a l .{ }^{20}$ They reviewed studies published between 2000 and 2005, on adherence to treatment for diabetes, hypertension and dyslipidemia, revealing that only $51 \%$ of patients treated with LLT were having medication on hand for more than $80 \%$ of their days on therapy after 12 months. ${ }^{20}$ Adherence studies in other populations than ours have shown that older age, concurrent medication, presence of CVD and other comorbidities such as type 2 diabetes, tend to increase adherence to statins, the most prescribed of LLT. $^{34}{ }^{35}$ In our fairly young cohort, 9\% had a history of CVD and $43 \%$ were treated with antihypertensive medication, which is reflected in the level of adherence where those with prior CVD and with other medications than insulin more often were among those with high adherence. In a study on adherence to statin guidelines in patients with type 1 diabetes and with over 50 years of diabetes duration, $72 \%$ of the patients selfreported adherence to statins, but fewer in primary than in secondary intervention, 68.5 vs $84.4 \%$ respectively. ${ }^{36}$ In the general population and in type 2 diabetes, adherence to statins tends to deteriorate over time and has been reported to be below $25 \%$ in high-risk patients after 5 years of treatment. ${ }^{14} 3738$ In our study of individuals with type 1 diabetes, 27\% had discontinued LLT after 18 months. Early diminishing use and discontinuation of LLT is unfortunate since high levels of adherence and longer duration of persistence with statins are associated with incremental improvement in clinical outcomes in patients at risk for cardiovascular events. ${ }^{13} 1439$

One reason why patients do not stay on treatment could be unwarranted side effects. In a large survey by Wei $e t a l$, perceived muscle pain was the far most common reason for nonpersistence accounting for $60 \%$ of discontinuation. ${ }^{40}$ In randomized controlled trials, serious adverse events from treatment with statins are sparse though, also when it comes to muscle-related problems, and when experienced side-effects from statins have been tested against placebo, there was no longer a significant difference between placebo and active substance. ${ }^{41-43}$ Claims of nonserious but symptomatic side effects can accordingly be a hindrance to treatment adherence and hence a cause for insufficient cardiovascular prevention. Healthcare professionals are important coactors in this. In a recent study from Italy, reasons for discontinuation of statins were assessed in 655 patients with suspected statin-induced adverse reactions and revealed that over $80 \%$ of statin discontinuations were due to adverse reactions classified as not serious, concluding that healthcare professionals' had a substantial impact on persistence to LLT, by discontinuing medication for not valid reasons. ${ }^{44}$

Perceived lack of efficacy can also be a reason for low adherence and discontinuation of statin therapy. ${ }^{40}$ One way to counteract perceptions like this could be to follow the recently published guideline on Cardiovascular Disease and Risk Management, from American Diabetes Association and the guideline on Management of Cholesterol from American College of Cardiology/ American Heart Association, updated in January 2018 and November 2018, respectively, and regularly assess LDL-cholesterol after initiation of statin therapy in order to increase adherence. ${ }^{45}{ }^{46}$ Some guidelines are now suggesting adding more pharmacological treatment to statins in order to reach LDL-cholesterol treatment targets in patients at high risk for CVD. If adherence to statin therapy was regularly scrutinized in clinical practice, treatment targets might be reached in more patients with statin treatment alone.

\section{Strengths and limitations}

There are several strengths of this study: first, the large sample of individuals with type 1 diabetes being novel users of LLT; second, the prospective approach and capacity to reflect usage in a real-world setting; third, our register-based study provides detailed data on cause of death, socioeconomic status, major comorbidities as well as risk factors including laboratory measures.

There are also limitations. In an observational study, there is always a possibility for residual confounding due to unmeasured variables, for example, hereditary factors and comorbidities such as psychiatric disease, which could influence both adherence and outcome. ${ }^{47}$ We may also have overestimated adherence as we do not know that all filled medications are consumed as prescribed, which would attenuate the association between adherence and CVD. Another bias to consider is the healthy adherer bias. High adherence could be a marker of healthy behavior and therefore a lower risk of a CVD event, even if we adjust for body mass index, physical activity and smoking as a proxy. Taking this into consideration, our results on lower CVD risk with high 
adherence could be overinterpreted. On the other hand, we excluded patients that could be of a certain fragility. Only patients who survived the exposure period were eligible for inclusion and we also excluded individuals with multidose dispensed medications and individuals with combination therapy, who are often older and with more concurrent diseases and with a higher risk for a cardiovascular event. These exclusions could lead to a selection bias with younger and somewhat healthier participants than in a real-life setting, hence underestimating the cardiovascular risk in our population and attenuating the impact of adherence.

\section{CONCLUSION}

This nationwide study among individuals with type 1 diabetes individuals and novel users of LLT shows that high refill adherence to LLT was associated with $22 \%$ lower risk for nonfatal CVD. Individuals discontinuing LLT within 18 months had a $43 \%$ higher risk of nonfatal CVD. It is important to evaluate and emphasize adherence to prescribed LLT at clinical visits in order to reach full potential of LLT and so reduce the risk of CVD in individuals with type 1 diabetes.

\section{Author affiliations}

${ }^{1}$ Department of Molecular and Clinical Medicine, Sahlgrenska Academy, University of Gothenburg, Gothenburg, Sweden

${ }^{2}$ Department of Medicine, Sahlgrenska University Hospital, Gothenburg, Sweden ${ }^{3}$ Department of Public Health and Community Medicine, Sahlgrenska Academy, University of Gothenburg, Gothenburg, Sweden

${ }^{4}$ National Diabetes Register, Centre of Registers, Västra Götaland, Gothenburg, Sweden

${ }^{5}$ Health Metrics Unit, Sahlgrenska Academy, University of Gothenburg, Gothenburg, Sweden

${ }^{6}$ Medical Evidence and Observational Research Centre, AstraZeneca AB, Gothenburg, Sweden

Acknowledgements The authors thank the regional National Diabetes Register (NDR) coordinators, as well as contributing nurses, physicians and patients.

Contributors Study concept and design: $\mathrm{CH}, \mathrm{SAK}, \mathrm{A}-\mathrm{MS}$, KAS, BE and KE-0. Statistical analyses: MM and SF. Interpretation of data: CH, SAK, KAS, A-MS, $\mathrm{BE}, \mathrm{KE}-0$, SF and SG. Drafting of manuscript: $\mathrm{CH}$ and KE-0. Critical revision and completion of manuscript by all authors. The manuscript has been read and approved by all coauthors.

Funding The study was financed by grants from the Swedish state under the agreement between the Swedish government and the county councils, the ALF agreement (ALFGBG-698991 and ALFGBG-430711) and from the Gothenburg Society of Medicine. The Swedish Diabetes Association and the Swedish Society of Diabetology support the NDR. The Swedish Association of Local Authorities and Regions funds the NDR.

Competing interests KAS is employed by AstraZeneca. However, the views expressed in this study are her own and not those of AstraZeneca. SG has received personal fees (lecture fees and research grants) from AstraZeneca, Boehringer Ingelheim, Eli Lilly, MSD, Novo Nordisk and Sanofi. KE-0 has received personal lecture fees from Eli Lilly and Abbott. BE has received personal fees for lectures and serving on advisory boards from Amgen, AstraZeneca, Boehringer Ingelheim, Eli Lilly, Merck Sharp \& Dohme, Mundipharma, Navamedic, Novo Nordisk and RLS Global and research grants from Sanofi. $\mathrm{CH}$ has received personal lecture fees from Eli Lilly.

Patient consent for publication Not required.

Provenance and peer review Not commissioned; externally peer reviewed.

Data availability statement No data are available. The data that support the findings of this study are not publicly available. The study presented here have been subject to an application to an ethical board and approved for publication related to the specific aim of our research project. With reference to the European General Data Protection Regulation (GDPR), the data are personal data and thereby protected by secrecy. Study definitions and descriptive statistics are available on request.

Open access This is an open access article distributed in accordance with the Creative Commons Attribution Non Commercial (CC BY-NC 4.0) license, which permits others to distribute, remix, adapt, build upon this work non-commercially, and license their derivative works on different terms, provided the original work is properly cited, appropriate credit is given, any changes made indicated, and the use is non-commercial. See: http://creativecommons.org/licenses/by-nc/4.0/.

ORCID iDs

Christel Hero http://orcid.org/0000-0001-7090-8952

Sofia Axia Karlsson http://orcid.org/0000-0002-1896-9587

Katarina Eeg-Olofsson http://orcid.org/0000-0002-3376-4707

\section{REFERENCES}

1 Krolewski AS, Kosinski EJ, Warram JH, et al. Magnitude and determinants of coronary artery disease in juvenile-onset, insulindependent diabetes mellitus. Am J Cardiol 1987:59:750-5.

2 Livingstone SJ, Levin D, Looker $\mathrm{HC}$, et al. Estimated life expectancy in a Scottish cohort with type 1 diabetes, 2008-2010. JAMA 2015;313:37-44.

3 Rawshani A, Sattar N, Franzén S, et al. Excess mortality and cardiovascular disease in young adults with type 1 diabetes in relation to age at onset: a nationwide, register-based cohort study. Lancet 2018;392:477-86.

4 Soedamah-Muthu SS, Chaturvedi N, Toeller M, et al. Risk factors for coronary heart disease in type 1 diabetic patients in Europe: the EURODIAB prospective complications study. Diabetes Care 2004:27:530-7.

5 Lind M, Svensson A-M, Kosiborod M, et al. Glycemic control and excess mortality in type 1 diabetes. $N$ Engl $J$ Med 2014;371:1972-82.

6 Baigent C, Keech A, Kearney PM, et al. Efficacy and safety of cholesterol-lowering treatment: prospective meta-analysis of data from 90,056 participants in 14 randomised trials of statins. Lancet 2005;366:1267-78.

7 Heart Protection Study Collaborative Group. MRC/BHF heart protection study of cholesterol lowering with simvastatin in 20,536 high-risk individuals: a randomised placebo-controlled trial. Lancet 2002;360:7-22.

8 Kearney PM, Blackwell L, Collins R, et al. Efficacy of cholesterollowering therapy in 18,686 people with diabetes in 14 randomised trials of statins: a meta-analysis. Lancet 2008;371:117-25.

9 Hero C, Rawshani A, Svensson A-M, et al. Association between use of lipid-lowering therapy and cardiovascular diseases and death in individuals with type 1 diabetes. Diabetes Care 2016;39:996-1003.

10 World Health Organization. Adherence to long-term therapies: evidence for action.

11 Chowdhury R, Khan H, Heydon E, et al. Adherence to cardiovascular therapy: a meta-analysis of prevalence and clinical consequences. Eur Heart J 2013;34:2940-8.

12 Ruokoniemi P, Sund R, Arffman M, et al. Are statin trials in diabetes representative of real-world diabetes care: a population-based study on statin initiators in Finland. BMJ Open 2014:4:e005402.

13 Simpson RJ, Mendys P. The effects of adherence and persistence on clinical outcomes in patients treated with statins: a systematic review. J Clin Lipidol 2010;4:462-71.

14 Perreault S, Dragomir A, Blais L, et al. Impact of better adherence to statin agents in the primary prevention of coronary artery disease. Eur J Clin Pharmacol 2009;65:1013-24.

15 Korhonen MJ, Ruokoniemi P, llomäki J, et al. Adherence to statin therapy and the incidence of ischemic stroke in patients with diabetes. Pharmacoepidemiol Drug Saf 2016;25:161-9.

16 Karlsson SA, Hero C, Svensson A-M, et al. Association between refill adherence to lipid-lowering medications and the risk of cardiovascular disease and mortality in Swedish patients with type 2 diabetes mellitus: a nationwide cohort study. BMJ Open 2018;8:e020309.

17 Scaldaferri F, Pizzoferrato M, Ponziani FR, et al. Use and indications of cholestyramine and bile acid sequestrants. Intern Emerg Med 2013;8:205-10.

18 Osterberg L, Blaschke T. Adherence to medication. N Engl J Med 2005;353:487-97. 
19 Lesén E, Sandström TZ, Carlsten A, et al. A comparison of two methods for estimating refill adherence to statins in Sweden: the rare project. Pharmacoepidemiol Drug Saf 2011;20:1073-9.

20 Cramer JA, Benedict Á., Muszbek N, et al. The significance of compliance and persistence in the treatment of diabetes, hypertension and dyslipidaemia: a review. Int $J$ Clin Pract 2008;62:76-87.

21 Deshpande S, Quek RGW, Forbes CA, et al. A systematic review to assess adherence and persistence with statins. Curr Med Res Opin 2017;33:769-78.

22 Karlsson SA, Hero C, Eliasson B, et al. Refill adherence and persistence to lipid-lowering medicines in patients with type 2 diabetes: a nation-wide register-based study. Pharmacoepidemiol Drug Saf 2017;26:1220-32.

23 Eliasson B, Gudbjörnsdottir S. Diabetes care--improvement through measurement. Diabetes Res Clin Pract 2014;106(Suppl 2):S291-4.

24 Wettermark B, Hammar N, MichaelFored C, et al. The new Swedish prescribed drug Register-Opportunities for pharmacoepidemiological research and experience from the first six months. Pharmacoepidemiol Drug Saf 2007;16:726-35.

25 Ludvigsson JF, Andersson E, Ekbom A, et al. External review and validation of the Swedish national inpatient register. BMC Public Health 2011;11:450.

26 Ingelsson E, Arnlöv J, Sundström J, et al. The validity of a diagnosis of heart failure in a hospital discharge register. Eur J Heart Fail 2005;7:787-91.

27 Statistics Sweden. Longitudinal integration database for health insurance and labour market studies (LISA by Swedish acronym). Available: http://www.scb.se/lisa-en/ [Accessed Last accessed Sep 2019].

28 Friedewald WT, Levy RI, Fredrickson DS. Estimation of the concentration of low-density lipoprotein cholesterol in plasma, without use of the preparative ultracentrifuge. Clin Chem 1972:18:499-502.

29 Levey AS, Bosch JP, Lewis JB, et al. A more accurate method to estimate glomerular filtration rate from serum creatinine: a new prediction equation. modification of diet in renal disease Study Group. Ann Intern Med 1999;130:461.

30 Zhang Q, Safford M, Miller D, et al. Short-term statin exposure is associated with reduced all-cause mortality in persons with diabetes. Med Care 2007;45:308-14.

31 De Vera MA, Bhole V, Burns LC, et al. Impact of statin adherence on cardiovascular disease and mortality outcomes: a systematic review. Br J Clin Pharmacol 2014;78:684-98.

32 Collins R, Armitage J, Parish S, et al. MRC/BHF heart protection study of cholesterol-lowering with simvastatin in 5963 people with diabetes: a randomised placebo-controlled trial. Lancet 2003:361:2005-16.

33 Ruokoniemi P, Korhonen MJ, Helin-Salmivaara A, et al. Statin adherence and the risk of major coronary events in patients with diabetes: a nested case-control study. $\mathrm{Br} J$ Clin Pharmacol 2011;71:766-76.
34 Perreault S, Blais L, Dragomir A, et al. Persistence and determinants of statin therapy among middle-aged patients free of cardiovascular disease. Eur J Clin Pharmacol 2005;61:667-74.

35 Helin-Salmivaara A, Lavikainen P, Ruokoniemi P, et al. Persistence with statin therapy in diabetic and non-diabetic persons: a nationwide register study in 1995-2005 in Finland. Diabetes Res Clin Pract 2009;84:e9-11.

36 Bai JW, Boulet G, Halpern EM, et al. Cardiovascular disease guideline adherence and self-reported statin use in longstanding type 1 diabetes: results from the Canadian study of longevity in diabetes cohort. Cardiovasc Diabetol 2016;15:14.

37 Donnelly LA, Doney ASF, Morris AD, et al. Long-term adherence to statin treatment in diabetes. Diabet Med 2008;25:850-5.

38 Toth PP, Granowitz C, Hull M, et al. Long-term statin persistence is poor among high-risk patients with dyslipidemia: a real-world administrative claims analysis. Lipids Health Dis 2019;18:175.

39 Law MR, Wald NJ, Thompson SG. By how much and how quickly does reduction in serum cholesterol concentration lower risk of ischaemic heart disease? BMJ 1994;308:367-72.

40 Wei MY, Ito M, Cohen J, et al. Predictors of statin adherence, switching, and discontinuation in the usage survey: understanding statin use in America and gaps in patient Education*. J Clin Lipidol 2013;7:245-6.

41 Collins R, Reith C, Emberson J, et al. Interpretation of the evidence for the efficacy and safety of statin therapy. Lancet 2016;388:2532-61.

42 Gupta A, Thompson D, Whitehouse A, et al. Adverse events associated with unblinded, but not with blinded, statin therapy in the Anglo-Scandinavian cardiac outcomes Trial-Lipid-Lowering arm (ASCOT-LLA): a randomised double-blind placebo-controlled trial and its non-randomised non-blind extension phase. Lancet 2017;389:2473-81.

43 Newman CB, Preiss D, Tobert JA, et al. Statin safety and associated adverse events: a scientific statement from the American heart association. Arterioscler Thromb Vasc Biol 2019;39:e38-81.

44 Sessa M, Rafaniello C, Scavone C, et al. Preventable statin adverse reactions and therapy discontinuation. what can we learn from the spontaneous reporting system? Expert Opin Drug Saf 2018;17:457-65.

45 American Diabetes Association. 9. Cardiovascular disease and risk management: standards of medical care in diabetes-2018. Diabetes Care 2018;41:S86-104.

46 Grundy SM, Stone NJ, Bailey AL, et al. 2018 AHA/ACC/AACVPR/ AAPA/ABC/ACPM/ADA/AGS/APhA/ASPC/NLA/PCNA guideline on the management of blood cholesterol: a report of the American College of Cardiology/American heart association task force on clinical practice guidelines. J Am Coll Cardiol 2019;73:e285-350.

47 DiMatteo MR, Lepper HS, Croghan TW. Depression is a risk factor for noncompliance with medical treatment: meta-analysis of the effects of anxiety and depression on patient adherence. Arch Intern Med 2000;160:2101-7. 